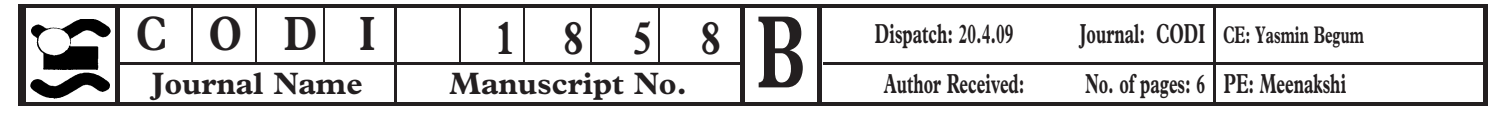

\title{
Suppression of the postoperative neutrophil leucocytosis following neoadjuvant chemoradiotherapy for rectal cancer and implications for surgical morbidity
}

\author{
S. F. Kerr*, M. Klonizakis† and R. Glynne-Jonesł \\ *Department of Surgery, Barnet Hospital, Barnet, Hertfordshire, †East of England Research Design Service, University of Hertfordshire, Hertfordshire and
} ‡Centre for Cancer Treatment, Mount Vernon Hospital, Northwood, Middlesex, UK

Received 27 November 2008; accepted 13 January 2009

\begin{abstract}
Objective The extent to which neoadjuvant chemoradiotherapy for rectal cancer influences postoperative morbidity is controversial. This study investigated whether this treatment suppresses the normal perioperative inflammatory response and explored the clinical implications.
\end{abstract}

Method Prospective databases were queried to identify 37 consecutive study patients undergoing definitive surgery following 5 -FU/capecitabine-based chemoradiotherapy and 35 consecutive untreated control patients operated upon for rectal or rectosigmoid cancer. Preoperative ( $<10$ days) and postoperative $(<24 \mathrm{~h})$ neutrophil counts, along with morbidity data, were confirmed retrospectively. Univariate and multivariate analyses assessed the apparent effect of chemoradiotherapy on change in neutrophil count. The latter's association with postoperative morbidity was then examined.

Results Sufficient data were available for 34 study and 27 control patients. Repeated-measures ANCOVA revealed significant differences between their perioperative neutrophil counts $(P=0.02)$. Of the other characteristics which differed between the groups, only age and tumour location were prognostically significant regarding perioperative change in neutrophil count. Accounting for relevant covariates, chemoradiotherapy was significantly associated with a suppressed perioperative neutrophil leucocytosis. Local postoperative complications affected 25 of 61 patients, who had lower perioperative neutrophil increases than their counterparts $(P=0.016)$.

Conclusion Chemoradiotherapy appears to suppress the perioperative inflammatory response, thereby increasing susceptibility to local postoperative complications.

Keywords Rectal cancer, neoadjuvant chemoradiotherapy, surgery, postoperative complications, immunosuppression, leucocyte

\section{Introduction}

Meticulous surgical dissection along embryological planes represents the mainstay of treatment for resectable rectal cancer, but significant recurrence rates with surgery alone and advances in imaging techniques have contributed to the rationale for preoperative therapy. Neoadjuvant radiotherapy has been demonstrated to improve local control and overall survival and the addition of chemotherapy reduces local recurrence further [1].

Correpondence to: R. Glynne-Jones, Centre for Cancer Treatment, Mount Vernon Hospital, Rickmansworth Road, Northwood, Middlesex HA6 2RN, UK. E-mail: rob.glynnejones@nhs.net
Short-course radiotherapy, delivered over 1 week and followed by surgery a few days later, increases postoperative morbidity [2-5]. The extent to which this also applies to chemoradiotherapy, typically administered over 5 weeks and followed by surgery several weeks later, is more controversial. It is theoretically plausible that the combined myelosuppressive effects of chemotherapy and pelvic radiotherapy attenuate the normal inflammatory response to surgical trauma, thereby increasing susceptibility to complications. Objective evidence of immunosuppression at the time of surgery following chemoradiotherapy for rectal cancer has been reported previously, but any correlation with clinical morbidity was not investigated [6]. However, among patients treated with short-course radiotherapy, a suppressed 
postoperative neutrophil leucocytosis appears to predict postoperative morbidity [7].

The purpose of this study was to investigate whether the normal postoperative neutrophil leucocytosis following chemoradiotherapy and delayed surgery is: (i) suppressed; (ii) associated with local postoperative complications; and (iii) related to the time interval to surgery.

\section{Method}

Extensive data on all rectal cancer patients treated with neoadjuvant chemoradiotherapy and definitive surgery at Barnet Hospital have been prospectively collected since 1994. The 37 most recently and consecutively treated patients were selected from this database as the study group. A control group of 34 consecutive patients undergoing elective surgery for cancer of the rectum or rectosigmoid without neoadjuvant therapy was identified from a second prospectively compiled database of all colorectal cancer patients diagnosed since 2006. The use of all data for the purposes of this study was approved by the local research ethics committee.

For all patients, local staging was based on a combination of clinical assessment and pelvic magnetic resonance imaging (MRI), while distant metastases were excluded by computed tomography (CT) of abdomen and thorax. Treatment was recommended to patients in light of discussion by a multidisciplinary team, comprising surgeons, oncologists, radiologists and pathologists. Eligibility criteria for neoadjuvant chemoradiotherapy included histologically confirmed rectal adenocarcinoma involving or threatening the anticipated resection margin, World Health Organisation (WHO) performance status 0-2 and acceptable haematological and renal function, i.e. neutrophils $>1.5 \times 10^{9} / 1$, platelets $\geq 100 \times 10^{9} / 1$ and serum creatinine $<1.25$ times the institution's upper limit of normal range.

Megavoltage external beam radiotherapy was delivered using a three- or four-field box technique in the prone position using CT-based three-dimensional planning. To derive a planning target volume, margins were added to the gross tumour volume as follows: $3 \mathrm{~cm}$ laterally, superiorly and inferiorly; $2 \mathrm{~cm}$ anteriorly. The posterior border was always located on the most posterior aspect of the bony sacrum. All study patients received a total of $45 \mathrm{~Gy}$, delivered in 25 daily fractions of $1.8 \mathrm{~Gy}$ over 33 days.

Concurrent chemotherapy was in the form of 5 fluorouracil (5-FU) or its oral equivalent capecitabine. The former was usually administered as a 60 -min infusion at a dose of $350 \mathrm{mg} / \mathrm{m}^{2}$, along with $20 \mathrm{mg} / \mathrm{m}^{2}$ folinic acid, on each of the first five and last 5 days of radiotherapy. Alternatively, a prolonged venous infusion of 5 -FU was administered at a daily dose of $200 \mathrm{mg} / \mathrm{m}^{2}$ for the entire duration of radiotherapy and for 3 weeks beyond. Capecitabine was prescribed at a twice daily dose of $850 \mathrm{mg} / \mathrm{m}^{2}$ during each day of radiotherapy.

Local protocol recommended that surgery be scheduled for 6-12 weeks following completion of radiotherapy. In practice, patients were usually assessed at 6 weeks by the operating surgeon, who decided whether to allow additional time for tumour shrinkage. Surgery was performed by, or under the direct supervision of, one of four consultant surgeons with colorectal expertise, who routinely carried out total mesorectal excision.

Surgical specimens were examined grossly and microscopically using TNM criteria, according to the Royal College of Pathologists' minimum dataset for colorectal cancer [8]. All lymph nodes retrieved were examined histologically and any extramural deposits larger than $3 \mathrm{~mm}$ in diameter were considered to be nodal. Patients were usually reviewed by the surgical team within 6 weeks of their operation. For the purposes of this study, complete wound healing was defined as intact skin with no evidence of erythema, tenderness, fluctuance, discharge or a sinus. Anastomotic leakage was diagnosed on the basis of intra-operative findings, or clinically directed imaging alone if laparotomy was not performed.

Neutrophil counts were obtained retrospectively from the hospital's laboratory computer system. Preoperative counts were defined as having been collected within 10 days prior to surgery, while postoperative neutrophil counts were all collected on the first postoperative day. The incidences of local postoperative complications, namely delayed perineal wound healing, anastomotic leakage and re-laparotomy for any other reason, were confirmed by retrospective review of discharge letters and outpatient correspondence. Patients were excluded from subsequent analysis if neutrophil counts were not available from these specific times or if sufficient details of their postoperative recovery could not be obtained.

Data were analysed using statistical software (SPSS 2 version 15, SPSS, USA). Shapiro-Wilks tests assessed the degree of normality for all baseline and outcome variables. Depending on these, chi-square tests (for categorical variables) and Mann-Whitney U-test, ANOVA or unpaired $t$-tests (for continuous data) were used to assess differences between the chemoirradiated and untreated patients. Effect sizes were measured using Cohen's $d$ or Cramer's $\varphi$, depending on the variable type and the comparison test used [9]. Correlation and equivalency tests were used to study the prognostic value of baseline variables in predicting changes in neutrophil count and complications. Repeated-measures ANCOVA models were developed incorporating relevant covariates 
to study the association between chemoradiotherapy and change in neutrophil count. Similarly, ANCOVA models were developed to study the association between change in neutrophil count and postoperative complications. The association between change in neutrophil count and interval length was assessed using unpaired $t$-tests.

\section{Results}

Sufficient data on perioperative neutrophil counts and morbidity were available for 34 of 37 study patients and 27 of 34 control patients. Among the study patients, 19 had received oral capecitabine and 15 had received intravenous 5-FU, of which the prolonged regimen throughout the entire duration of radiotherapy and for 3 weeks beyond was administered to only two patients.

One study patient was treated for recurrent rectal cancer; all other patients had primary disease. One patient in each group underwent simultaneous cystoprostatectomy and urinary diversion, while two study and one control patients underwent hysterectomy and bilateral salpingo-oophorectomy. Other baseline, operative and pathological characteristics for each of the groups are shown in Table 1. Both preoperative and postoperative neutrophil counts were approximately normally distributed.

Figure 1 portrays the perioperative change in neutrophil count for each group. Correlation and equivalency tests determined that age, tumour distance from anal verge and chemoradiotherapy were the only prognostically significant variables for this outcome. Although the two groups' preoperative neutrophil counts were similar, repeated-measures ANCOVA revealed significant differences between study and control patients $(P=0.02)$, with a marked effect size $\left(\eta^{2}=0.14\right)$ and observed power $(0.85)$. When relevant covariates were incorporated in this 'between-subjects' comparison, neither age nor tumour distance from anal verge had significant effects

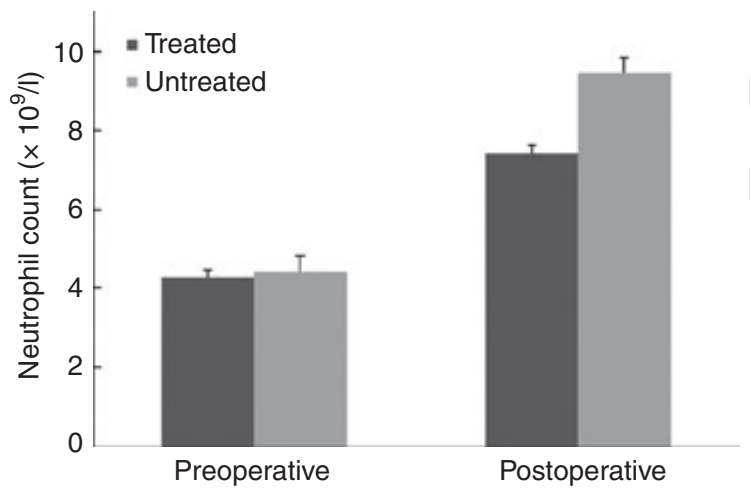

Figure I Perioperative neutrophil counts.

(Table 2). Figure 2 portrays the perioperative increases in neutrophil count in terms of estimated marginal means, i.e. means after the covariates have been accounted for.

'Within-subjects' comparison tests confirmed a significant postoperative increase in neutrophil count, irrespective of group (Table 3 ). The interaction of measurement points with the variables included in the model did not reveal any significant differences for age or distance, whereas chemoradiotherapy did appear to be a major determinant of this increase (Figure 3).

Four patients died prior to discharge, including three within 30 post-operative days; all four had experienced anastomotic leakage. In total, seven of 26 anterior resections were complicated by clinically apparent anastomotic leakage. Two other patients with small bowel obstruction, one with intra-abdominal haemorrhage and one with a small intestinal fistula required re-laparotomy prior to discharge. Complete perineal healing was not achieved by 6 weeks following 16 of 30 abdominoperineal resections.

In total, 25 of $61(41.0 \%)$ patients experienced at least one of these local postoperative complications. Their preoperative neutrophil counts were similar to those of
Table I Baseline, operative and pathological characteristics.

\begin{tabular}{lllll}
\hline & $\begin{array}{l}\text { Study } \\
(n=34)\end{array}$ & $\begin{array}{l}\text { Control } \\
(n=27)\end{array}$ & $\begin{array}{l}P \text {-value } \\
\text { Male }\end{array}$ & $\begin{array}{l}\text { Effect } \\
\text { size* }\end{array}$ \\
Age (years) & 19 & 20 & 0.14 & 0.19 \\
Distance from anal verge $(\mathrm{cm})$ & $62.3 \pm 2.1$ & $73.6 \pm 2.1$ & 0.004 & 0.84 \\
AR:APR:Hartmann's & $4.3 \pm 0.2$ & $11.0 \pm 0.9$ & $<0.001$ & 1.62 \\
Laparoscopic & $6: 26: 2$ & $20: 4: 3$ & $<0.001$ & 0.86 \\
(y)T3-4 & 0 & 8 & $<0.001$ & 0.43 \\
Preop neutrophil count $\left(\times 10^{9} / 1\right)$ & 18 & 20 & 0.09 & 0.70 \\
\hline
\end{tabular}

Continuous data presented as mean \pm SE.

${ }^{*}$ Calculated as Cohen's $d$ and Cramer's $\varphi$, for continuous and categorical variables, respectively. 
Table 2 Tests of 'between-subjects' effects ( ${ }^{*}$ computed using alpha $=0.05$ ).

\begin{tabular}{llrlll}
\hline Source & df & $F$ & $P$-value & $\eta^{2}$ & $\begin{array}{l}\text { Observed } \\
\text { power* }\end{array}$ \\
\hline Intercept & 1 & 35.608 & 0.000 & 0.39 & 1.00 \\
Distance & 1 & 0.825 & 0.367 & 0.02 & 0.15 \\
Age & 1 & 0.037 & 0.848 & 0.001 & 0.05 \\
Chemoradiotherapy & 1 & 6.255 & 0.015 & 0.10 & 0.69 \\
\hline
\end{tabular}

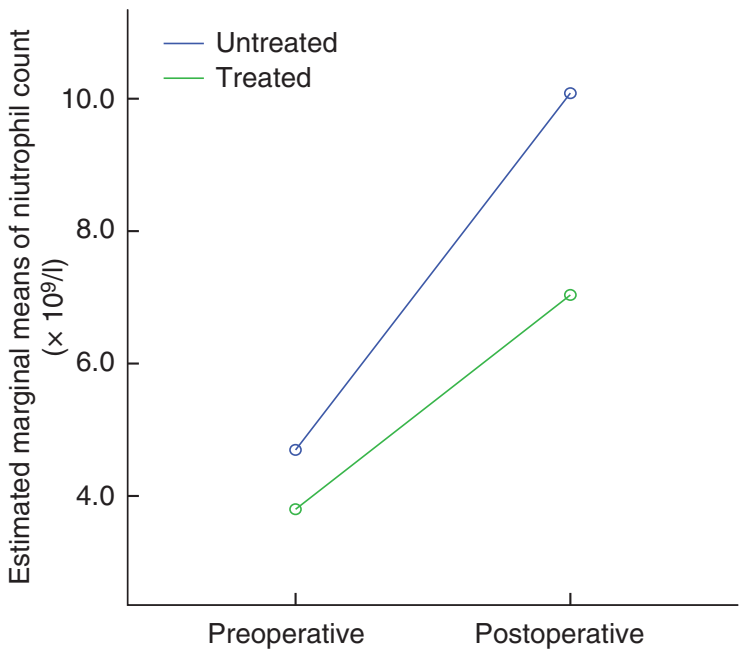

Figure 2 Estimated marginal means of perioperative neutrophil counts.

Table 3 Tests of 'within-subjects' effects ( ${ }^{*}$ computed using alpha $=0.05$ ).

\begin{tabular}{llrlll}
\hline Source & df & $F$ & $P$-value & $\eta^{2}$ & $\begin{array}{l}\text { Observed } \\
\text { power* }\end{array}$ \\
\hline Neutrophil increase & 1 & 11.641 & 0.001 & 0.17 & 0.92 \\
Distance & 1 & 0.172 & 0.680 & 0.003 & 0.07 \\
Age & 1 & 2.009 & 0.162 & 0.04 & 0.29 \\
Chemoradiotherapy & 1 & 6.236 & 0.015 & 0.10 & 0.69 \\
\hline
\end{tabular}

their 36 counterparts, with means of $4.5 \times 10^{9} / 1$ and $4.2 \times 10^{9} / 1$ respectively $(P=0.348)$. Mean perioperative increases in neutrophil count were $3.0 \times 10^{9} / 1$ and $4.7 \times 10^{9} / 1$ respectively $(P=0.016)$. With ANCOVA, the association between change in neutrophil count and complications failed to reach statistical significance $(P=0.09)$, although the effect size was moderate $\left(\eta^{2}=0.05\right)$,

For the 34 study patients, the median interval from completion of radiotherapy to surgery was 74.5 (range 50-118) days. This value was used to divide these

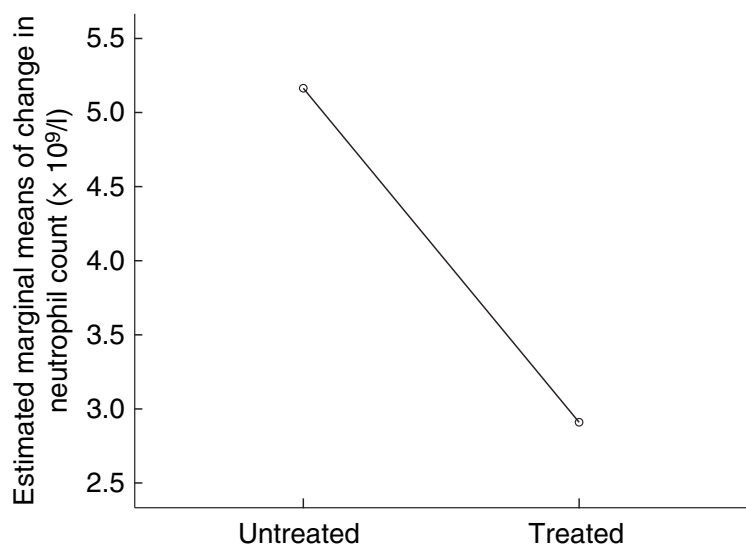

Figure 3 Estimated marginal means of change in neutrophil count.

patients into two equally sized groups. Mean preoperative neutrophil count was $4.3 \times 10^{9} / 1$ for the short interval group and $4.2 \times 10^{9} / 1$ for the long interval group $(P=0.747)$. Mean perioperative increases in neutrophil count were $3.1 \times 10^{9} / 1$ and $3.2 \times 10^{9} / 1$ respectively $(P=0.905)$.

\section{Discussion and conclusions}

At present, it is difficult to determine from the literature the extent to which neoadjuvant chemoradiotherapy for rectal cancer influences postoperative morbidity. Most of the large randomized trials of short-course radiotherapy have demonstrated a detrimental effect of this regimen, particularly with respect to perineal wound healing [2-5]. In turn, three randomized trials of preoperative chemoradiotherapy $v s$ short-course radiotherapy have reported that the rates of postoperative complications were not significantly different between the two regimens [10-12]. Combined modality treatment has also been implicated as a cause of surgical morbidity by some retrospective analyses [13-15]. By contrast, the only completed randomized trial of preoperative $v s$ postoperative chemoradiotherapy reported no significant difference in postoperative morbidity between the two groups [16]. However, it is possible that a true detrimental effect of chemoradiotherapy per se was obscured by the fact that this treatment caused down-staging and altered the operative strategy in some patients.

This study provides evidence that neoadjuvant chemoradiotherapy for rectal cancer suppresses the normal inflammatory response to surgical trauma, as measured by the postoperative neutrophil leucocytosis, even at a median of 11 weeks following treatment completion. This is broadly consistent with Wichmann et al.'s prospective study, in which various inflammatory and 
immunological markers were suppressed perioperatively among 15 rectal cancer patients treated with chemoradiotherapy in comparison with 15 matched controls [6]. Their discrepant finding that preoperative granulocyte count also differed significantly between the two groups may be due to the fact that their patients proceeded to surgery within a shorter interval of 4-6 weeks. Differences in the perioperative changes in granulocyte count between the two groups were not reported in their study and the implications for clinical morbidity were not investigated.

This study also found evidence of a suppressed postoperative neutrophil leucocytosis among those patients subsequently developing local postoperative complications in comparison with their counterparts who had uncomplicated recoveries. This resembles Hartley et al.'s experience with short-course preoperative radiotherapy [7]. Although this secondary association lost statistical significance on multivariate analysis, this may simply reflect the small sample size, particularly as there was a moderate effect size. A larger series of patients would be required to explore satisfactorily the clinical significance of any myelosuppressive effect of chemoradiotherapy.

The findings of any small study such as this should be interpreted with caution, as it is possible that they could have arisen by chance. Much of the principal data were collected retrospectively and, although consecutive patients were recruited to minimize selection bias, some were subsequently excluded due to incomplete data. Perhaps its main limitation is that the study and control patients were not optimally matched: for example, the former were younger and had lower rectal tumours and two factors which would have contributed to their selection for neoadjuvant therapy. However, the statistical analysis suggests that the apparent effect of chemoradiotherapy is unlikely to be attributable to any of the other observed differences between the groups.

Although pathological tumour stage was similar between the groups, the study patients might have had considerably larger tumours prior to treatment, which one might speculate could have contributed to immunosuppression. It could also be argued that each of the patient groups was rather heterogeneous: for example, all the study patients did not receive exactly the same chemotherapeutic regimen. A few patients underwent synchronous resection of adjacent organs, but they were at least evenly distributed between the groups. The single measurements of wound healing and postoperative neutrophil count are rather crude outcome data, albeit consistently collected. Finally, the relatively high rate of anastomotic leakage may simply reflect the small sample size and has broad confidence intervals.
It might have been expected that the chemoradiotherapy-induced suppression of the postoperative neutrophil leucocytosis would have become less pronounced with longer intervals to surgery, as the bone marrow recovered. This could have provided one explanation for the positive association between shorter intervals and postoperative morbidity reported recently by the authors [17]. Although no such influence of interval length was apparent in this study, a definitive exploration of this hypothesis would require a larger series of patients.

In summary, neoadjuvant chemoradiotherapy is increasingly employed in the management of rectal cancer but its implications for surgical morbidity remain controversial. Consistent with the limited available evidence, this study suggests that the normal perioperative inflammatory response is suppressed by this treatment, increasing susceptibility to local postoperative complications. Further research is needed to ensure that patients with rectal cancer derive the optimal long-term oncological benefit of chemoradiotherapy at minimal cost to their prospects of an uncomplicated postoperative recovery.

\section{Acknowledgements}

This study was based on a prospectively-compiled database, to which the contributions of Ms Juliet Grainger and the Rob Glynne-Jones Research Fund are acknowledged. We are also grateful for the assistance of $\mathrm{Mr} \mathrm{R}$ Harrison, Mr I Mitchell, Mr P Mathur and Mr C Elton, of Barnet Hospital.

\section{References}

1 Wong RKS, Tandan V, De Silva S, Figueredo A. Preoperative radiotherapy and curative surgery for the management of localized rectal carcinoma. Cochrane Database Syst Rev 2007; Issue 2: Art No CD002102.

2 Marijnen CAM, Kapiteijn E, van de Velde CJH et al. Acute side effects and complications after short-term preoperative radiotherapy combined with total mesorectal excision in primary rectal cancer: report of a multicenter randomised trial. J Clin Oncol 2002; 20: 817-25.

3 Anonymous. Swedish Rectal Cancer Trial: Initial report from a Swedish multicentre study examining the role of preoperative irradiation in the treatment of patients with resectable rectal carcinoma. Br J Surg 1993; 80: 1333-6.

4 Pahlman L, Glimelius B. Pre- or postoperative radiotherapy in rectal and rectosigmoid carcinoma: report from a randomised multicenter trial. Ann Surg 1990; 211: 187-95.

5 Goldberg PA, Nicholls RJ, Porter NH, Love S, Grimsey JE. Long-term results of a randomised trial of short-course lowdose adjuvant pre-operative radiotherapy for rectal cancer: reduction in local treatment failure. Eur J Cancer 1994; 30A: 1602-6. 
6 Wichmann MW, Meyer G, Adam M et al. Detrimental immunologic effects of preoperative chemoradiotherapy in advanced rectal cancer. Dis Colon Rectum 2003; 46: 875-87.

7 Hartley A, Giridharan S, Srihari N, McConkey C, Geh JI. Impaired postoperative neutrophil leucocytosis and acute complications following short course preoperative radiotherapy for operable rectal cancer. Eur J Surg Oncol 2003; 29: 155-7.

8 Royal College of Pathologists Working Group on Cancer Services. (1998) Dataset for Colorectal Cancer Histopathology Reports. Royal College of Pathologists, London.

9 Cohen J. (1988) Statistical Power Analysis for the Behavioural Sciences. Erlbaum, Hillsdale, NJ.

10 Bosset J-F, Collette L, Calais G et al. Chemotherapy with preoperative radiotherapy in rectal cancer. $N$ Engl J Med 2006; 355: 1114-23.

11 Gerard J-P, Conroy T, Bonnetain F et al. Preoperative radiotherapy with or without concurrent fluorouracil and leucovorin in T3-4 rectal cancers: results of FFCD 9203. J Clin Oncol 2006; 24: 4620-5.

12 Bujko K, Nowacki MP, Kepka L, Oledzki J, Bebenek M, Kryj M. Postoperative complications in patients irradiated pre- operatively for rectal cancer: report of a randomised trial comparing short-term radiotherapy vs chemoradiation. Colorectal Dis 2005; 7: 410-6.

13 Buie WD, Maclean AR, Attard JP, Brasher PMA, Chan AK. Neoadjuvant chemoradiation increases the risk of pelvic sepsis after radical excision of rectal cancer. Dis Colon Rectum 2005; 48: 1868-74.

14 Bullard KM, Trudel JL, Baxter NN, Rothenberger DA. Primary perineal wound closure after preoperative radiotherapy and abdominoperineal resection has a high incidence of wound failure. Dis Colon Rectum 2005; 48: 438-43.

15 Artioukh DY, Smith RA, Gokul K. Risk factors for impaired healing of the perineal wound after abdominoperineal resection of rectum for carcinoma. Colorectal Dis 2006; 9: 362-7

16 Sauer R, Becker H, Hohenberger W et al. Preoperative versus postoperative chemoradiotherapy for rectal cancer. $N$ Engl J Med 2004; 351: 1731-40.

17 Kerr SF, Norton S, Glynne-Jones R. Delaying surgery after neoadjuvant chemoradiotherapy for rectal cancer may reduce postoperative morbidity without compromising prognosis. Br J Surg 2008; 95: 1534-40. 


\section{Author Query Form}

\section{Journal: $\quad$ CODI}

Article: $\quad 1858$

Dear Author,

During the copy-editing of your paper, the following queries arose. Please respond to these by marking up your proofs with the necessary changes/additions. Please write your answers on the query sheet if there is insufficient space on the page proofs. Please write clearly and follow the conventions shown on the attached corrections sheet. If returning the proof by fax do not write too close to the paper's edge. Please remember that illegible mark-ups may delay publication.

Many thanks for your assistance.

\begin{tabular}{|l|l|l|}
\hline $\begin{array}{l}\text { Query } \\
\text { reference }\end{array}$ & Query & Remarks \\
\hline 1 & $\begin{array}{l}\text { AUTHOR: A running head short title was not supplied; please check } \\
\text { if this one is suitable and, if not, please supply a short title of up to } 40 \\
\text { characters that can be used instead. }\end{array}$ & \\
\hline 2 & AUTHOR: Please give address information for 'SPSS': town and state. & \\
\hline
\end{tabular}




\section{Please correct and return this set}

Please use the proof correction marks shown below for all alterations and corrections. If you wish to return your proof by fax you should ensure that all amendments are written clearly in dark ink and are made well within the page margins.

\begin{tabular}{|c|c|c|}
\hline Instruction to printer & Textual mark & Marginal mark \\
\hline Leave unchanged & ... under matter to remain & ( ) \\
\hline $\begin{array}{l}\text { Insert in text the matter } \\
\text { indicated in the margin }\end{array}$ & $h$ & $\begin{array}{l}\text { New matter followed by } \\
h \text { or } h \otimes\end{array}$ \\
\hline Delete & $\begin{array}{l}\text { I through single character, rule or underline } \\
\text { or }\end{array}$ & $\sigma$ or $\sigma(x)$ \\
\hline $\begin{array}{l}\text { Substitute character or } \\
\text { substitute part of one or } \\
\text { more word(s) }\end{array}$ & I through letter or & $\begin{array}{l}\text { new character / or } \\
\text { new characters / }\end{array}$ \\
\hline Change to italics & — under matter to be changed & $\leftarrow$ \\
\hline Change to capitals & $\equiv$ under matter to be changed & $\equiv$ \\
\hline Change to small capitals & $=$ under matter to be changed & $=$ \\
\hline Change to bold type & $\sim$ under matter to be changed & $\sim$ \\
\hline Change to bold italic & $\bar{\sim}$ under matter to be changed & $\underline{s i n}$ \\
\hline Change to lower case & Encircle matter to be changed & $\Rightarrow$ \\
\hline Change italic to upright type & (As above) & \\
\hline Change bold to non-bold type & (As above) & \\
\hline Insert 'superior' character & $\begin{array}{l}/ \text { through character or } \\
K \text { where required }\end{array}$ & $\begin{array}{l}y^{\prime} \text { or } y \\
\text { under character } \\
\text { e.g. } y^{2} \text { or } y^{2}\end{array}$ \\
\hline Insert 'inferior' character & (As above) & $\begin{array}{l}\lambda \\
\text { over character } \\
\text { e.g. } \hat{\Sigma}\end{array}$ \\
\hline Insert full stop & (As above) & $\odot$ \\
\hline Insert comma & (As above) & , \\
\hline Insert single quotation marks & (As above) & $\begin{array}{l}\dot{y} \text { or } \dot{x} \text { and/or } \\
\dot{y} \text { or } \dot{y}\end{array}$ \\
\hline Insert double quotation marks & (As above) & $\begin{array}{l}\ddot{y} \text { or } \ddot{y} \text { and/or } \\
\ddot{y} \text { or } \ddot{y}\end{array}$ \\
\hline Insert hyphen & (As above) & 1 \\
\hline Start new paragraph & 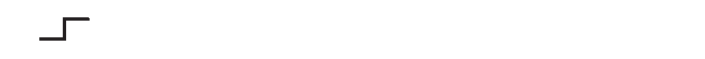 & 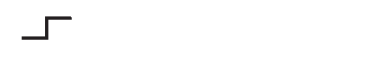 \\
\hline No new paragraph & $\infty$ & $\omega$ \\
\hline Transpose & $\sqcup$ & $\sqcup$ \\
\hline Close up & linking $\bigcirc$ characters & \\
\hline $\begin{array}{l}\text { Insert or substitute space } \\
\text { between characters or words }\end{array}$ & $\begin{array}{l}\text { I through character or } \\
\Lambda \text { where required }\end{array}$ & \\
\hline $\begin{array}{l}\text { Reduce space between } \\
\text { characters or words }\end{array}$ & $\begin{array}{l}\text { between characters or } \\
\text { words affected }\end{array}$ & $\uparrow$ \\
\hline
\end{tabular}

